Morgan Adams*, Ian Campbell, Cathy McCullagh, Donnacha Russell, Detlef W. Bahnemann, and Peter K.J. Robertson

\title{
From Ideal Reactor Concepts to Reality: The Novel Drum Reactor for Photocatalytic Wastewater Treatment
}

\begin{abstract}
This article reports the development of a novel drum photocatalytic reactor for treating dye effluent streams. The parameters for operation including drum rotation speed, light source distance, catalyst loading and $\mathrm{H}_{2} \mathrm{O}_{2}$ doping have been investigated using methylene blue as a model pollutant. Effluent can be generated by a number of domestic and industrial sources, including pharmaceutical, oil and gas, agricultural, food and chemical sectors. The work reported here proposes the application of semiconductor photocatalysis as a final polishing step for the removal of hydrocarbons from effluents sources, initial studies have proved effective in removing residual hydrocarbons from the effluent.
\end{abstract}

Keywords: drum photoreactor photocatalysis, mass transport, titanium dioxide $\left(\mathrm{TiO}_{2}\right)$, produced water

*Corresponding author: Morgan Adams, IDeaS, Innovation, Design and Sustainability Research Institute, Robert Gordon University, Schoolhill, Aberdeen, AB10 1FR, UK, E-mail: m.adams1@rgu.ac.uk Ian Campbell: E-mail: fitlyke@gmail.com, Cathy McCullagh: E-mail: c.mccullagh@rgu.ac.uk,

Donnacha Russell: E-mail: m.adams@rgu.ac.uk, IDeaS, Innovation, Design and Sustainability Research Institute, Robert Gordon University, Schoolhill, Aberdeen, AB10 1FR, UK

Detlef W. Bahnemann, Institut fuer Technische Chemie, Gottfried Wilhelm Leibniz Universitaet Hannover, Callinstrasse 3, D-30167 Hannover, Germany, E-mail: bahnemann@iftc.uni-hannover.de Peter K.J. Robertson, IDeaS, Innovation, Design and Sustainability Research Institute, Robert Gordon University, Schoolhill, Aberdeen, AB10 1FR, UK, E-mail: peter.robertson@rgu.ac.uk

\section{Introduction}

\subsection{Photocatalytic water treatment}

The application of photocatalysis for water purification has become a widely researched topic. As a water treatment technique, this process has several advantages as it destroys pollutants rather than merely transferring them to another phase (e.g. activation carbon adsorption, gas sparging) and does so without the use of potentially hazardous oxidants (e.g. ozone, chlorination) [1]. In order to fully assess the potential of photocatalysis as a water purification method, consideration needs to be given to the reactor unit in which the photocatalyst is deployed. There are a number of issues that need to be considered when designing a reactor to be employed for the photocatalytic purification of water [2]. A key parameter influencing the water treatment capability of the photocatalytic reactor is the quantity of catalyst that can be activated [3]. A major challenge in the development of effective photocatalytic reactors is, therefore, the efficient exposure of the catalyst to irradiation. Maximising the surface area of catalyst exposed to irradiation will increase the remediation potential of the photocatalytic reactor [4]. It is also imperative to ensure that the irradiated catalyst area in contact with the water stream is maximised, which can be achieved by ensuring efficient mixing within the system. Another significant issue is the effective mass transfer of the pollutants to the catalyst surface. In order to improve the destruction rate, the mass transfer of the process needs to be maximised, which will improve the interaction between the pollutant molecules and the catalyst. In order to allow for future industrial application, a high wastewater throughput through the reactor should also be achieved [5-7].

Typically applications utilising photocatalytic reactors for treating polluted water exhibit the difficulty of handling fluids having varying composition and/or concentrations; thus, a detailed kinetic representation may not be possible. To compare different reacting systems under similar operating conditions and to provide approximate estimations for scaling up purposes, simplified models may be useful. For these approximations, the model parameters should be restricted as much as possible to initial physical and boundary conditions such as initial concentrations (expressed as such or as TOC measurements), flow rate or reactor volume, irradiated reactor area, incident radiation fluxes and a fairly simple experimental observation such as the photonic efficiency. This article reports the development of a novel rotating drum reactor for the treatment of dye effluents. 


\section{Materials and methods}

\subsection{Materials}

Methylene blue (MB), purity: 85\% (remaining 15\% primarily) salt, was purchased from Aldrich and used in aqueous solution (Milli Q water). Titanium dioxide $\left(\mathrm{TiO}_{2}\right)$ in pellet form was purchased from Sachtleben Chemie, Duisburg, Germany (Hombikat KO1) and used as purchased; the composition was reported to contain $80 \%$ anatase and $20 \%$ rutile; specific surface area (BET) $95 \mathrm{~m}^{2} \mathrm{~g}^{-1}$, primary particle size $\sim 15 \mathrm{~nm}$, mean pore diameter $\left(\mathrm{N}_{2}\right) \sim 150 \AA$ A $\mathrm{pH} 5.5$. Degussa $\mathrm{P} 25 \mathrm{TiO}_{2}$ was used as received.

\subsection{Photocatalytic reactors}

\subsubsection{Reactor design 1}

The initial design of reactor 1 was created with agitation baffles to create a high level of interaction between the wastewater and catalyst suspension during rotation of the reactor drum. Figure 1 illustrates the first design of the drum reactor supported on rubber wheels with a plastic insert. The reactor drum was $350 \mathrm{~mm}$ in length, $150 \mathrm{~mm}$ diameter and 4 longitudinally positioned full length baffles. The wheel was mounted to brackets by means of a standard nut and bolt. Illumination was provided by $16 \times 8 \mathrm{~W}$ Philips T5 UV tubes (128 W total) supplied by RS Components Ltd, Northants, UK.

There was also an increase in friction generated at the running surfaces due to the external force exerted on the drum by the drive belt from the motor. The drive belt was elasticised generating compression forces hence

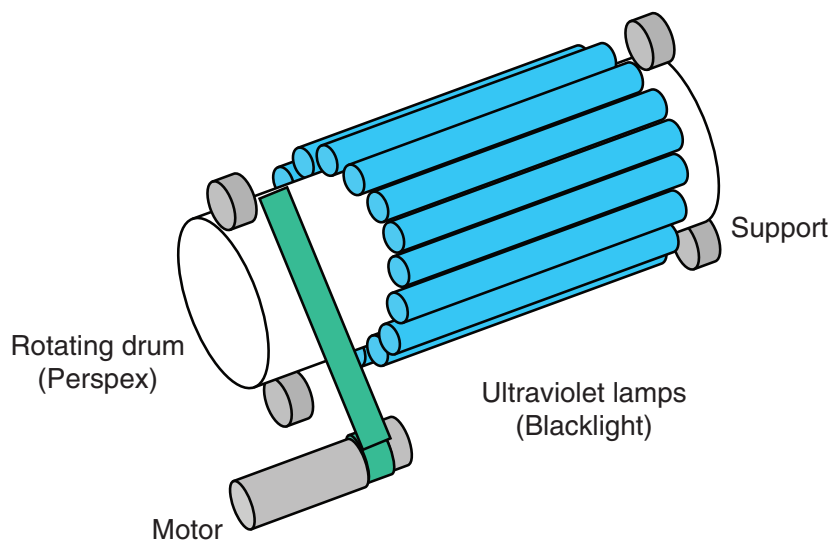

Figure 1 Reactor 1 schematic reproduced from [8] with permission.

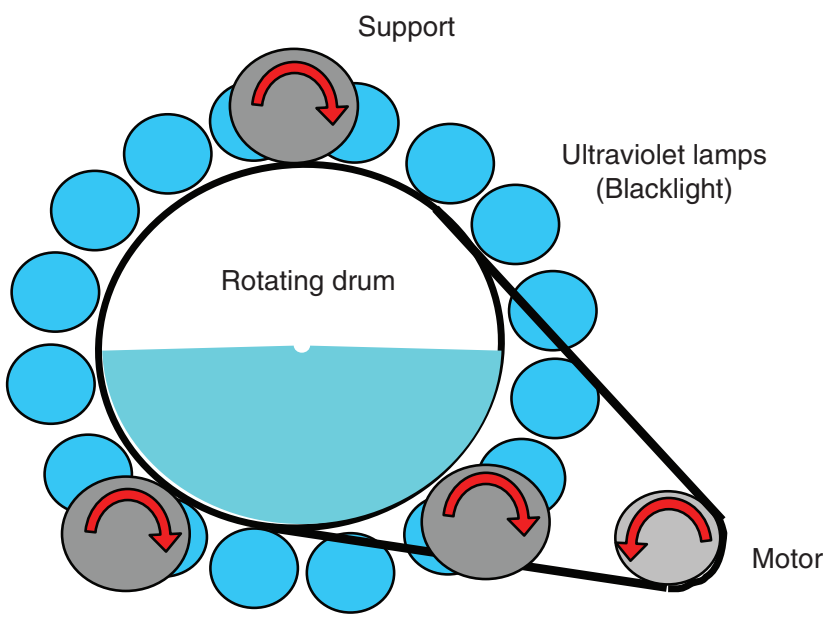

Figure 2 End elevation view of reactor 1 [8].

'pulling' on the drum (Figure 2). There were potential issues associated with friction highlighted and this led to a redesign of the test rig. A direct drive between the motor using frictionless bearings was designed allowing a reduction in friction and therefore reducing the torque required by the motor to rotate the drum, see Figure 3.

\subsubsection{Reactor design 2}

The design of the reactor was assessed in order to enhance agitation between the wastewater and catalyst suspension during rotation of the reactor drum. Having considered the method of agitation from reactor design 1 , and examining the mixing within a cement mixer, a series of "paddles" fixed to the inside of the mixing drum were designed and incorporated. On rotation of the drum, agitation in the fluid was caused by the movement of the paddles through that solution, resulting in a turbulent flow within the fluid. With the generation of turbulent flow, the mass transport of the pollutants to the catalyst surface was enhanced within the reactor unit. Figure 3 illustrates the first design of the drum reactor supported on rubber wheels with a plastic insert. The reactor drum was $400 \mathrm{~mm}$ in length with a diameter of $150 \mathrm{~mm}$ diameter. The wheel was mounted to brackets by means of a standard nut and bolt. Illumination was provided by $16 \times 8 \mathrm{~W}$ Philips T5 UV tubes (128 W total) supplied by RS Components Ltd, Northants, UK.

The design of the paddle system was a spiral pattern of paddle sections offset by $90^{\circ}$ between the first and the last section with an offset of $15^{\circ}$ between each section. This posed a problem in terms of placing the paddle sections at the correct increments and spacing. This was 


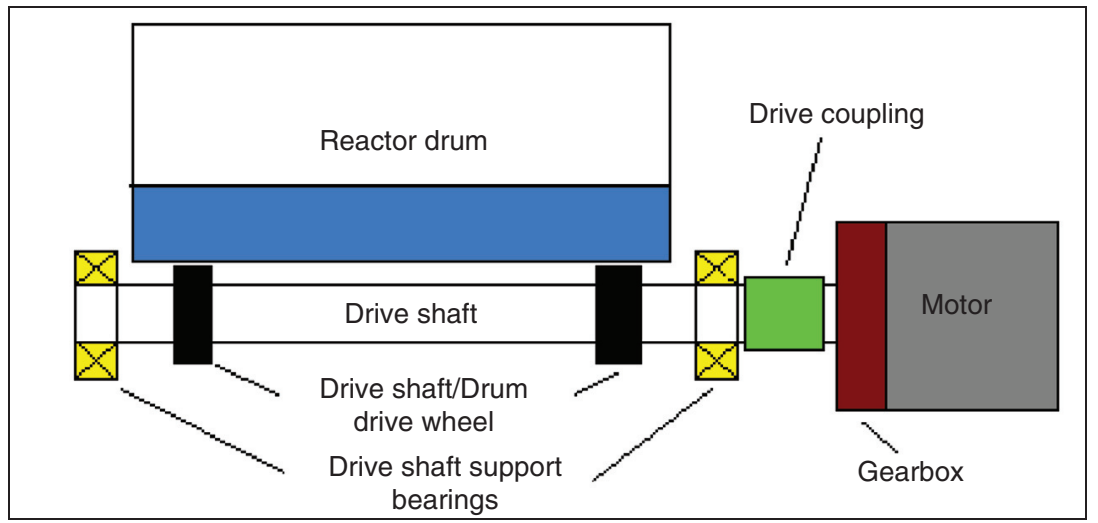

Figure 3 Diagram of the proposed redesign of the drive system.

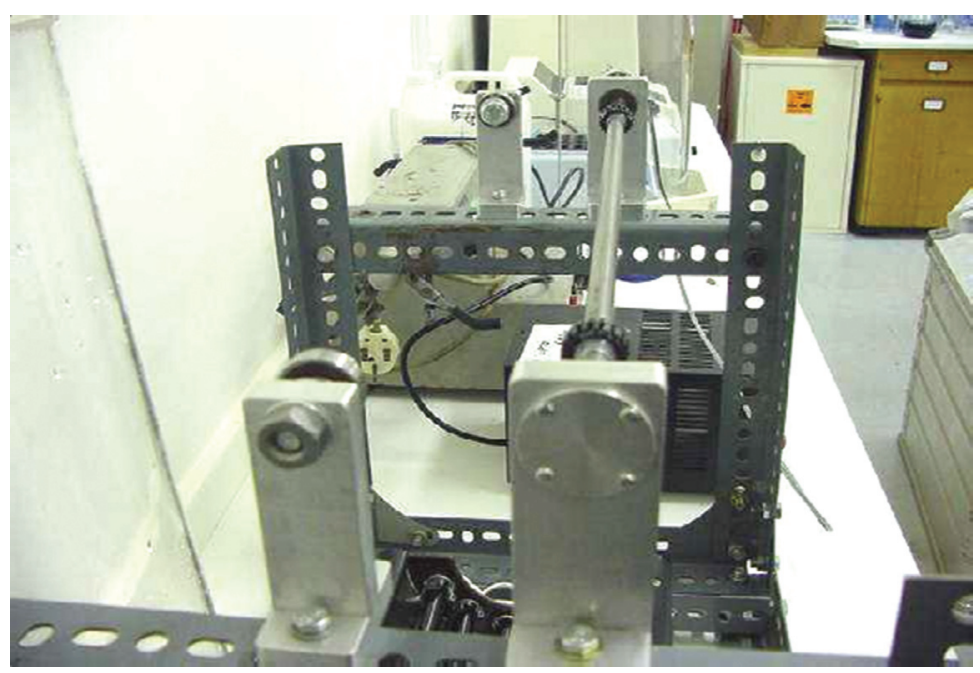

Figure 4 Photograph of the redesigned Test Rig fully assembled.

overcome by producing a template from tracing paper of the exact area of the inside of the drum cylinder. The positions in which the sections had to be placed were drawn on to the template. The template was then inserted into the cylinder and fixed in place. With the Perspex being transparent, the lines of where the sections were to be placed were visible from the outside. The outside of the cylinder was then marked with non-permanent marker, the template was removed and the sections were glued in place using Loctite superglue. The end caps were then glued in place and allowed to dry and the assembled reactor drum can be observed in Figure 4.

\subsubsection{Reactor design 3}

The third reactor design of the unit resulted in the development of a modular system capable of industrial application. The photocatalytic reactor consisted of: (i) three serially connected rotating cylindrical vessels (570 mm length and $94 \mathrm{~mm}$ i.d.) with weir-like paddles constructed along the longitudinal length of the vessels in a $\mathrm{V}$ arrangement

(ii) an external illumination source. Rotation of the cylindrical vessels was provided by three $12 \mathrm{dcV}$ electrical motors and illumination was provided by 36 W Philips PL-L sunlamp UV tubes supplied by RS Components Ltd, Northants, UK. The tubes were mounted in pairs, adjacent to each other on a reflective mirror, and enclosed in a wooden box to provide control over exposure to ambient light. The lamps were cooled by air flowing freely within the openings between the reactor vessels and the UV tubes.

Figure 5(a) and (b) show the patented photocatalytic drum reactor configuration $[8,9]$. Sampling was achieved via the open air vents at the fluid inlet side of the reactor drum. 


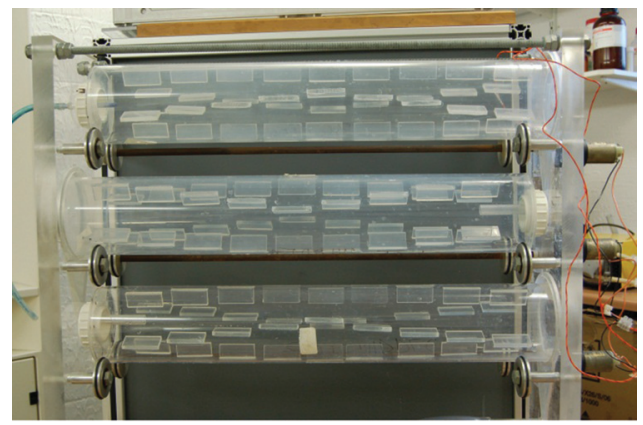

Plexiglas supports containing effluent

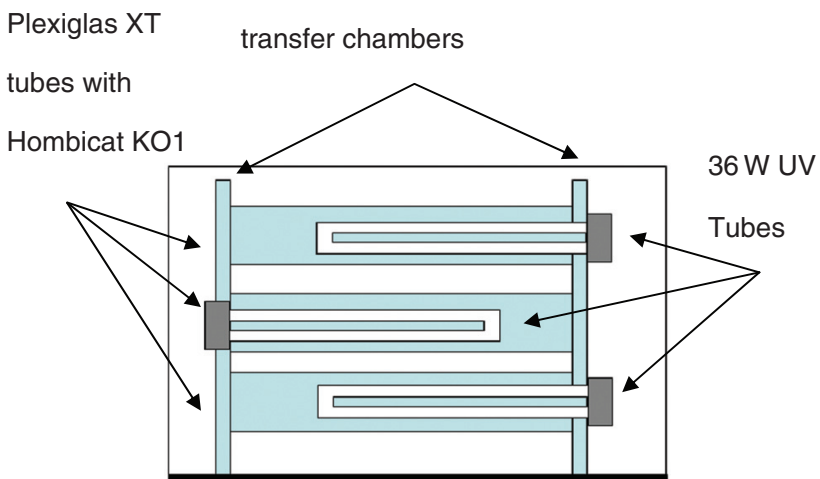

Figure 5 (a) Reactor 3 (b) Schematic of Reactor 3 configuration reproduced from [26] with permission from Elsevier.

\subsection{Photocatalytic experiments}

A stock solution of MB $(10 \mu \mathrm{M})$ was prepared and used for all experiments. The $\mathrm{TiO}_{2}$ pellet catalyst was weighed $(0.25 \mathrm{~g} 0.1 \% \mathrm{w} / \mathrm{v})$ and placed in the reaction vessel. To start the experiment, the feed solution was pumped (Autoclude Peristaltic pump) from the reservoir to the reaction vessel containing the catalyst. $\mathrm{MB}(10 \mu \mathrm{M}, 1 \mathrm{~L})$ was added into the reaction vessel containing the preweighed catalyst. The motor was switched on and the aqueous suspension continuously agitated for $60 \mathrm{~min}$ in the dark. Samples were drawn at 5 min intervals, centrifuged with Henderson T121 Centrifuge for $15 \mathrm{~min}$ at 6,000 rpm to eliminate any suspended $\mathrm{TiO}_{2}$ particles. The change in absorbance of $\mathrm{MB}$ was monitored at different time intervals using UV-visible spectroscopy (Perkin Elmer Lambda 950). Absorption spectra of samples were recorded between 200 and $750 \mathrm{~nm}$ and a maximum absorbance at $666 \mathrm{~nm}$ was used to calculate the concentration of MB. Prior to irradiation, the catalyst was immersed in distilled water for $60 \mathrm{~min}$ to enable effective hydration of the granular catalyst. The catalyst was then added to the MB solution in the drum reactor and photocatalysis was performed for $60 \mathrm{~min}$. UV illumination alone was also investigated. Samples were taken at 5 min intervals and treated as described previously.

\section{Results and discussion}

In order to optimise the reactor design assessment of the reactor performance was carried out using the following parameters: speed of rotation and light distance.

\subsection{Speed variation - reactor design 1}

The effect of rotational speeds of 15, 30, 45 and $60 \mathrm{rpm}$ was assessed by monitoring the decrease in absorbance of $\mathrm{MB}$

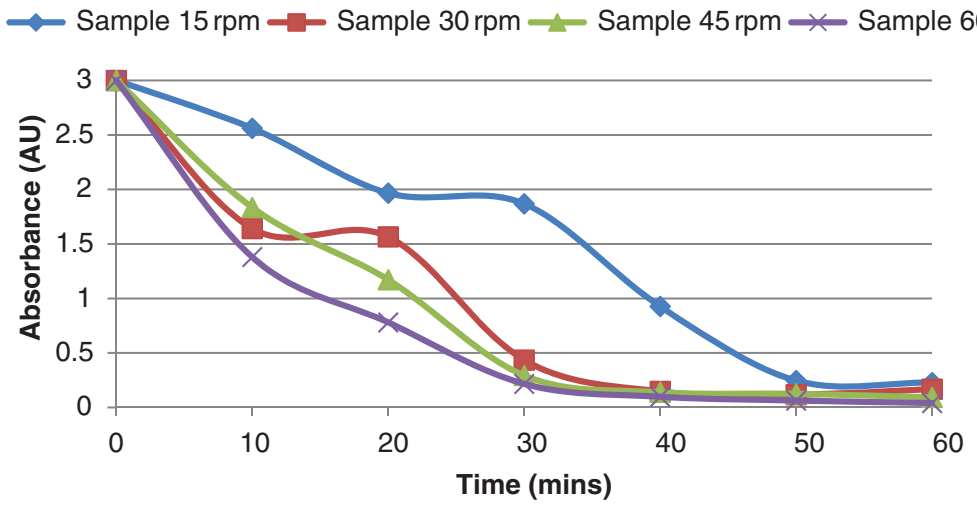

Figure 6 Effect of rotation speed on methylene blue degradation (version 1). 
following 60 minutes irradiation. Figure 6 illustrates the decrease in absorbance of $\mathrm{MB}$ at the four rotational speeds assessed. The optimum performance of the reactor was recorded at a speed of $60 \mathrm{rpm}$. At a rotational speed of $60 \mathrm{rpm}$, the solution was subjected to sufficient centrifugal force to deploy it consistently throughout the drum. A larger surface area of catalyst and solution was exposed to irradiation and an increase in turbulence was observed indicating that the reactor design was mass transport controlled. The plateauing in the breakdown of the dye observed at both $15 \mathrm{rpm}$ (between 20 and 30 minutes) and $30 \mathrm{rpm}$ (between 10 and 20 minutes) was most likely due to agglomeration of the catalyst due to inadequate dispersion through the dye effluent at these rotation speeds. As a result of the agglomeration of the catalyst, this would reduce to relative active area of the material and hence reduce the photocatalytic decomposition process.

\subsection{Light distance - reactor 1}

The positioning of the irradiation source is very important for any photocatalytic reactor in order to maximise the incident light intensity on the reactor surface and hence ensuring effective catalyst illumination. As previously reported, however, as light intensity increases, there is a linear increase in the photocatalytic reaction up to a certain level where the rate of reaction becomes proportional to the square root of the illuminating radiation intensity [10-13]. This is believed result from two effects. Firstly, at higher irradiation levels enhanced recombination of the electron/hole pair may occur
$[14,15]$. It has also been suggested that at high irradiation intensities surface-bound hydroxyl radicals may interact with one another in combination reactions rather than interacting with the substrate [16].

A series of light distances were investigated starting with a distance of $4 \mathrm{~cm}$, with the distance doubled for each subsequent experiment (Figure 7). The specifications are identical to that of the first set of experiments and the speed being used was the optimum speed. As Figure 7 shows there was an increased rate of $M B$ degradation from the closest lamp setting. Interestingly, there was not an even spread of degradation between 4, 8,16 and $32 \mathrm{~cm}$ which suggests the energy delivered from the lamp to the catalyst surface was not linear with sufficient delivery of photons up to $16 \mathrm{~cm}$. The end point for 4, 8 and $16 \mathrm{~cm}$ was within $10 \%$ of each other, so for the catalyst loading of $0.25 \mathrm{~g}$, the light distance was not critical over the full run of the decomposition reaction.

From the analysis of the results from the Drum reactor, an increase in rotational speed was found to increase mass transport which was expected. However, with speed increase, there is also an increase in the power required to operate the reactor thus making the process less cost effective.

\subsection{Drum reactor version 2}

The drum reactor version 2 was assessed using the same parameters as previously investigated, speed variation and light distance variation.

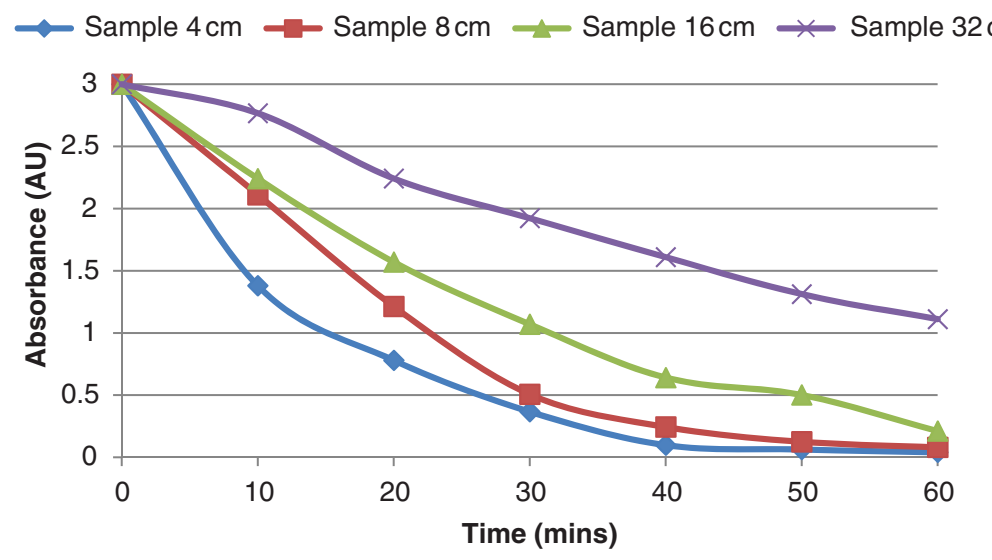

Figure 7 Effect of light distance on methylene blue degradation (version 1). 


\subsubsection{Optimum rotation speed - reactor 2}

The first experiment carried out involved an investigation on the variation of $\mathrm{MB}$ destruction rate due to the variation in rotational speed of the reactor. For this experiment, the distance between the UV lamps and the reactor drum was $4 \mathrm{~cm}$. The four speeds chosen were 15, 30, 45 and $60 \mathrm{rpm}$ and samples taken and analysed once the catalyst had settled out of solution. These results show that the optimum performance of the reactor occurs when the speed is $60 \mathrm{rpm}$ as with reactor design 1 (Figure 8).

As the photocatalytic process progress it could be seen that at $60 \mathrm{rpm}$, the MB solution tended to deploy itself more consistently around the drum walls. This is due to the friction caused between the solution and the drum wall and was also due to a small amount of centrifugal force. In turn this was likely to promote a larger surface area of solution/light. Furthermore, it also increased the turbulence within the solution thus increasing mass transport. These results indicate that mass transport is the rate determining step of this type of reactor design.

\subsubsection{Optimum light source distance - reactor 2}

In the second experiment, the destruction rate of $\mathrm{MB}$ was investigated with the UV lamps at various distances from the drum, 4, 8, 16 and $32 \mathrm{~cm}$. Doubling the distance between the UV lamps and the drum causes a fourfold reduction in the light intensity reaching the catalyst, i.e. it is a square root relationship.

The objective of investigating the effect of light intensity was to determine if the reactor was purely mass transport controlled or if other factors influence the rate of pollutant destruction and consider the influence of this with respect to the influence of mass transport as a rate determining factor with the reactor design. The experimental parameters were identical to that of the first experiment and the speed used was the optimum speed as detailed above (Figure 9).

As the optimum light distance was $4 \mathrm{~cm}$, the results are the same as that of the optimum speed because the speed variation experiments were conducted with a light at a distance of $4 \mathrm{~cm}$. Thus, the distance between the UV lamps and that reactor tube does affect the rate of the reaction.

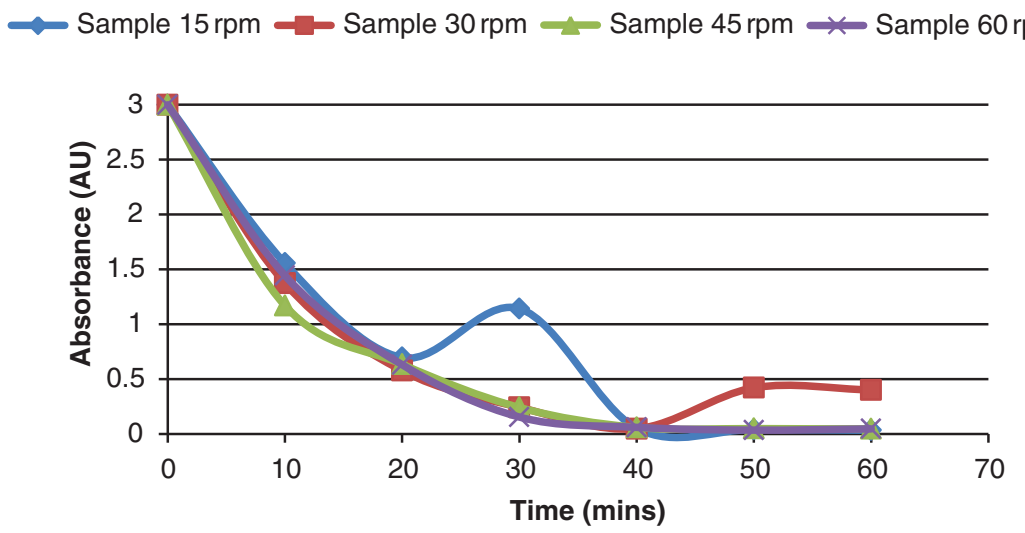

Figure 8 Effect of rotation speed on methylene blue degradation (version 2).

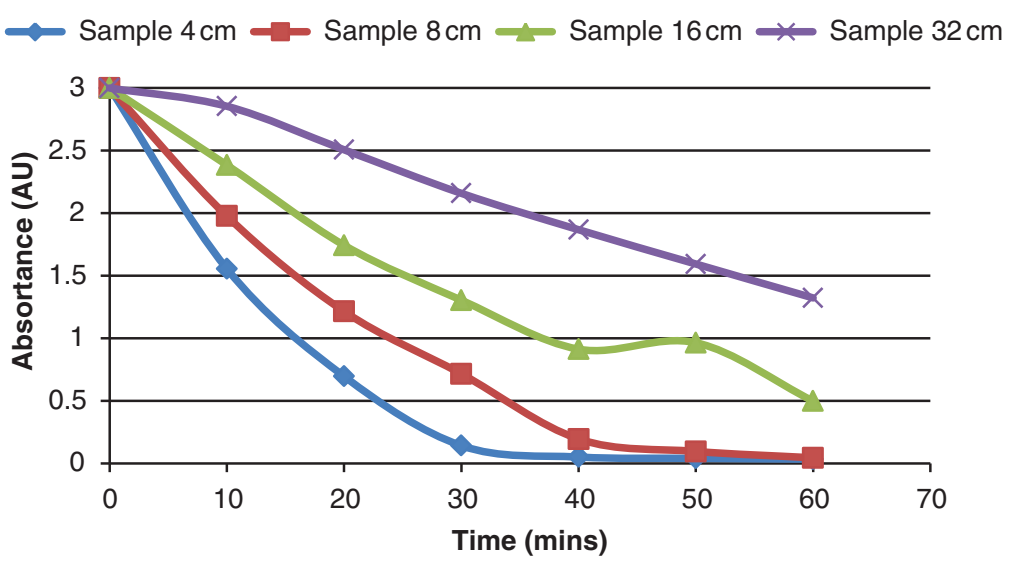

Figure 9 Effect of light distance on methylene blue degradation (version 2). 


\subsubsection{Hydrogen peroxide loading - reactor 2}

The addition of $\mathrm{H}_{2} \mathrm{O}_{2}$ can enhance the rate of photocatalytic processes through two mechanisms. Firstly, the peroxide can function as an alternative electron acceptor to oxygen (eq. 1). This process is thermodynamically more favourable reaction than oxygen reduction $[17,18]\left(E^{0}=\right.$ -0.13 for $\mathrm{O}_{2}$ reduction, $E^{0}=0.72$ for $\mathrm{H}_{2} \mathrm{O}_{2}$ reduction). Furthermore, the conductance band reduction of $\mathrm{H}_{2} \mathrm{O}_{2}$ will also generate hydroxyl radicals that will be available for attack on the substrate molecules [18]. As demonstrated by eq. (2), the peroxide may also react with superoxide generated by the reduction of oxygen at the conductance band, again producing hydroxyl radicals [19]. Consequently in the presence of $\mathrm{H}_{2} \mathrm{O}_{2}$ additional oxidising hydroxyl radicals may be generated in the system, no matter which conductance band reaction predominates, hence increasing the oxidising power of the system.

$$
\begin{gathered}
\mathrm{e}_{\mathrm{CB}}^{-}+\mathrm{H}_{2} \mathrm{O}_{2} \rightarrow \mathrm{OH}^{-}+\mathrm{OH} \\
\mathrm{O}_{2}^{-}+\mathrm{H}_{2} \mathrm{O}_{2} \rightarrow \mathrm{OH}^{-}+\mathrm{OH}+\mathrm{O}_{2}
\end{gathered}
$$

Four different concentrations of $\mathrm{H}_{2} \mathrm{O}_{2}$ were used -0.05 , $0.1,0.5$ and $1.0 \%(\mathrm{w} / \mathrm{v})$ respectively and for each experiment the UV light was set at a distance of $4 \mathrm{~cm}$ from the reactor vessel. On addition of $\mathrm{H}_{2} \mathrm{O}_{2}$, the dye solution started to degrade as soon as the catalyst was inserted in to the reactor (Figure 10).

From Figure 10, it can be seen that at higher concentrations of $\mathrm{H}_{2} \mathrm{O}_{2}$, initially greater initial rate of $\mathrm{MB}$ degradation was achieved with the majority of $\mathrm{MB}$ was degraded in the first 10 minutes of the experiment. As detailed above, this is likely to be due to the greater concentration of $\mathrm{H}_{2} \mathrm{O}_{2}$ is generating a greater number of $\mathrm{OH}$ radicals in the reactor which enhances the MB destruction. Even though the higher two peroxide concentrations initially caused a greater level of degradation, the overall breakdown values start overlapping between 10 and 15 minutes.

Consequently, it was concluded that the lowest percentage of $\mathrm{H}_{2} \mathrm{O}_{2}$ be chosen as the optimum loading for complete removal of the dye. It has previously been reported that higher peroxide concentrations can lower the destruction rate by competing for sites on the photocatalyst with the pollutant [20]. Also with the addition of $\mathrm{H}_{2} \mathrm{O}_{2}$, it was noted that the catalyst settled to the bottom of the solution far more rapidly than in the absence of $\mathrm{H}_{2} \mathrm{O}_{2}$. It is thought that the peroxide is influencing the surface charge on the catalyst and reducing its tendency to suspend.

\subsubsection{Effluent trial - reactor design 2}

To determine the overall breakdown of the hydrocarbons, and applicability of the drum reactor concept to industrial effluent streams, samples of "produced water" from the oil and gas sector were applied to the reactor. Table 1

Table 1 Chemical oxygen demand values after trial runs through reactor version 2 .

\begin{tabular}{lrrr}
\hline Sample & $\begin{array}{r}\text { Raw } \\
\text { effluent } \\
\text { COD mg/L }\end{array}$ & $\begin{array}{r}\text { 1st run through } \\
\text { reactor (version 2) } \\
\text { COD mg/L }\end{array}$ & $\begin{array}{r}\text { 2nd run through } \\
\text { reactor (version 2) } \\
\text { COD mg/L }\end{array}$ \\
\hline 1 & 3,490 & 2,000 & 720 \\
2 & 3,490 & 2,170 & 1,430 \\
3 & 3,620 & 2,180 & 400 \\
4 & 4,120 & 1,990 & 710 \\
\hline
\end{tabular}

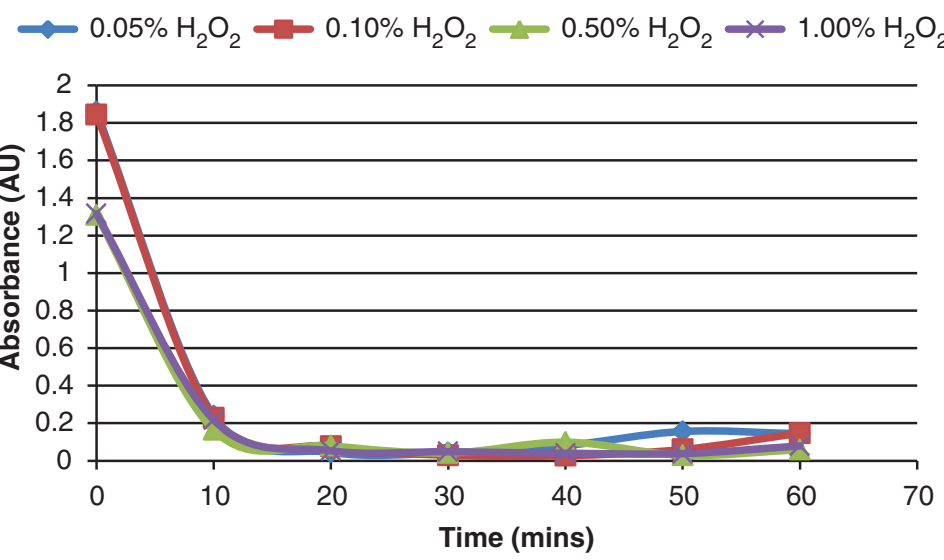

Figure 10 Absorbance at $664 \mathrm{~nm}$, for $\mathrm{H}_{2} \mathrm{O}_{2}$ at $0.05,0.1,0.5$ and $1.0 \%$. 
shows the chemical oxygen demand (COD) levels for 2 experimental runs.

From Table 1, it can be seen that even on one pass through the reactor there was between 40 and 50\% reduction in COD levels in the polluted water sample. On the second pass reduction levels of COD over $80 \%$ were attained.

\subsection{Drum reactor design 3}

The photocatalytic degradation of MB (Figure 11) was assessed as a model pollutant in reactor design 3. The rate of photodegradation was studied by monitoring the change in the maximum absorption in UV-visible spectra at $666 \mathrm{~nm}$ of the residual MB solution over irradiation time. Temporal changes in the concentration of MB were monitored by examining the changes in maximal absorption at $666 \mathrm{~nm}$ [21]. Absorbance of MB occurs typically at 668 and $609 \mathrm{~nm}$ [22]. The amount of residual MB decreased, while a blue shift of the maximum peak around 650-665 nm was observed, as the photocatalytic reaction proceeded, and this peak almost disappeared completely after 60 minutes of irradiation time (Figure 11). It was reported that the blue shift was caused by photo oxidative $\mathrm{N}$-demethylation of $\mathrm{MB}$ resulting from the production of intermediate species [23]. Prior to the experiment, the catalyst had been immersed in distilled water for $60 \mathrm{~min}$ utes. This was to ensure that the decrease in MB concentration observed resulted from photocatalysis and not adsorption of MB on to the catalyst surface. The quantity of $\mathrm{TiO}_{2}$ to be used for the experiment was placed in a beaker, and $500 \mathrm{~mL}$ of distilled water was added. When using new catalyst direct from the manufacturer, it was best to wash this way several times to remove excess surface powder from mechanical abrasion to ensure no suspended catalyst in the reactor.

Figure 12 also illustrates the degradation of $\mathrm{MB}$ in the presence of $0.1 \mathrm{gL}^{-1}$ of Degussa P25 catalyst. Following 20 minutes irradiation of $\mathrm{MB}, 90 \%$ remediation of $\mathrm{MB}$ was

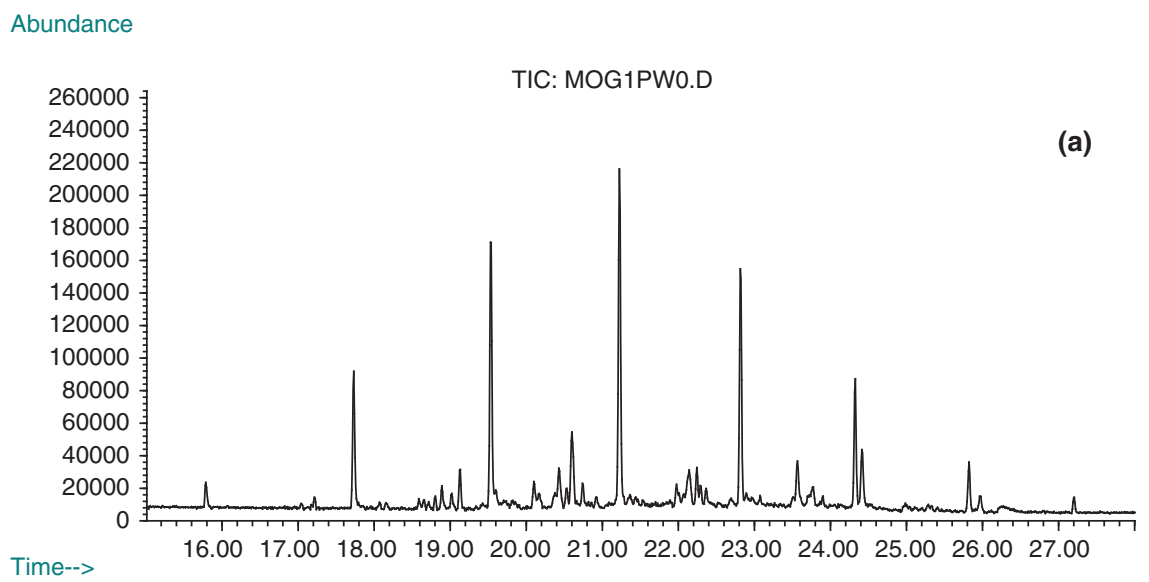

Abundance

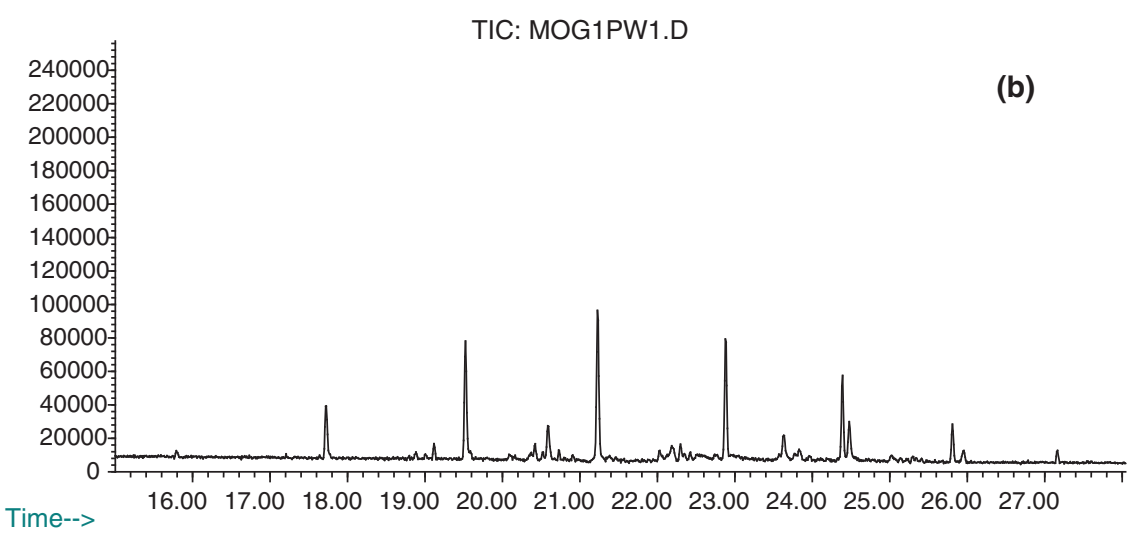

Figure 11 GC/MS Chromatogram of wastewater effluent (a) Pure sample with no treatment, (b) 1st pass through drum reactor, (c) 2nd pass through drum reactor and (d) 3rd pass through drum reactor (accumulative total) catalyst treatments over a 10 minutes irradiation. Source: Reproduced from [9] with permission from Hindawi. 
Abundance

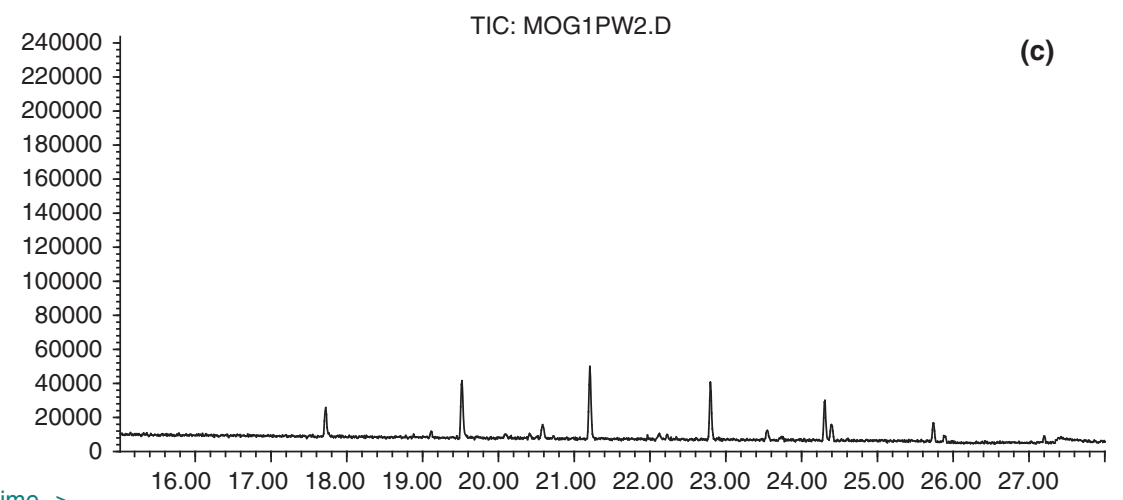

Time-->

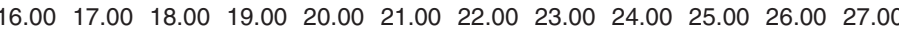

Abundance

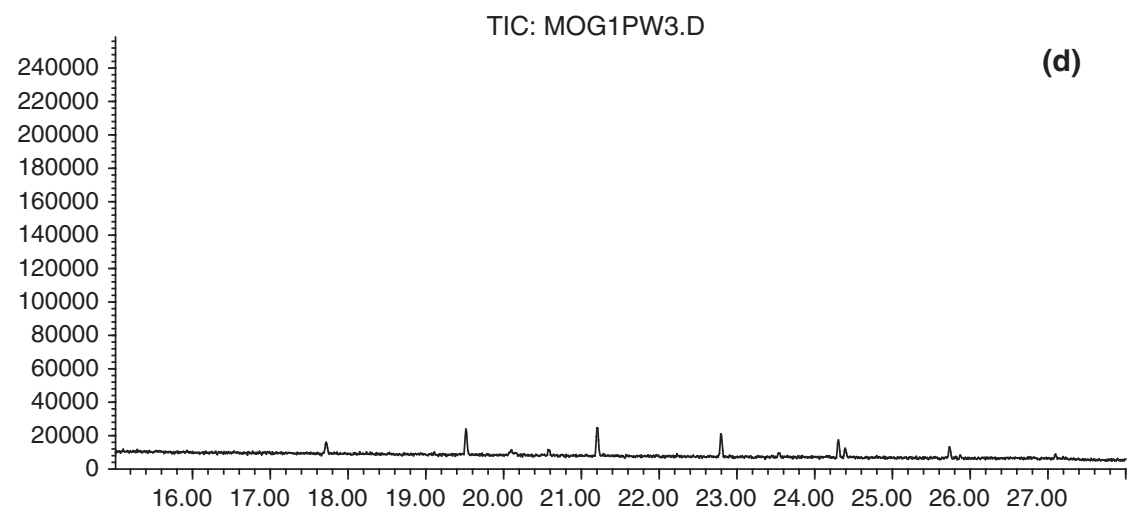

Time-->

Figure 11 (Continued)

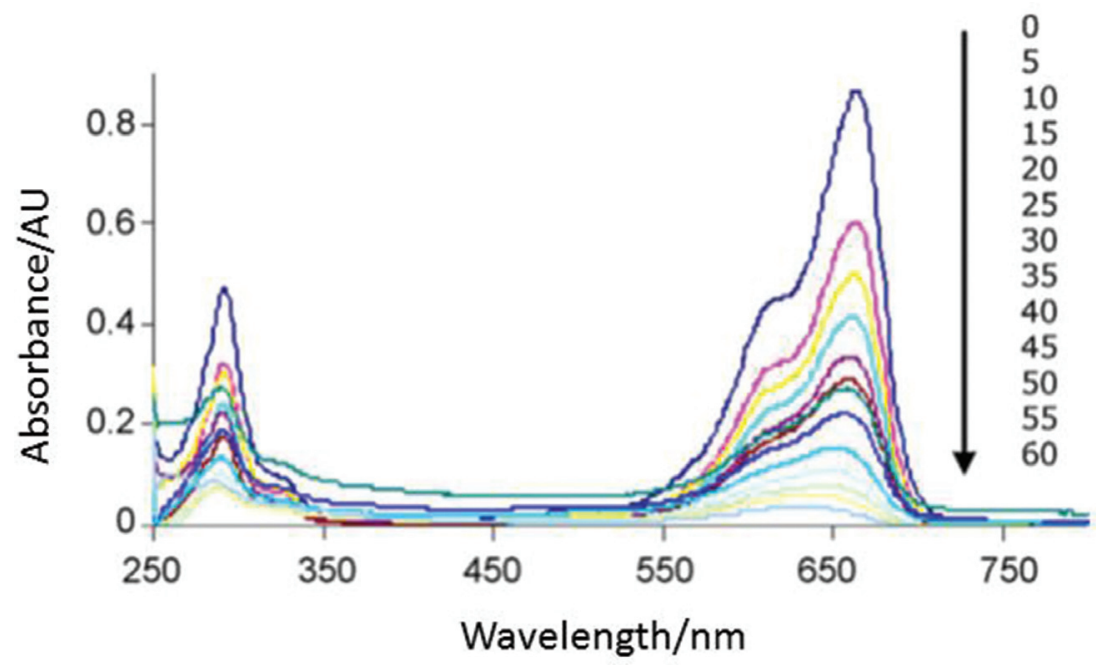

Figure 12 Blue shift around $665 \mathrm{~nm}$ caused by photooxidative N-demethylation of MB resulting from the production of intermediate species. Reproduced from [26] with permission from Elsevier. 
observed. This result demonstrates the inherent advantage of the reactor design. The patented baffles allow an increased surface area contact between catalyst and pollutant as well as high illumination of the catalyst. There have been many reports of reactors utilising Degussa P25 with various lamps for the remediation for MB ranging from $100 \%$ degradation of 20_M solution irradiated for 270 minutes with a $500 \mathrm{~W}$ Xenon lamp in the presence of $0.2 \mathrm{~g} \mathrm{~L}^{-1}$ catalyst [24]. Zhang et al. remediated a $0.1 \mathrm{mM}$ MB solution following 120 minutes irradiation from a $75 \mathrm{~W} \mathrm{Hg}$ lamp. The same group also reported the remediation of 25_M MB solution using a parabolic round concentrator reactor and solar illumination. This required 30 minutes to completely degrade MB [25].

The drum reactor was configured to be either a single pass or continuous flow system for produced water/effluents generated by the oil and gas sector. If after one pass, the water was still above the discharge level for hydrocarbons, the water was allowed to run into a lower reservoir. Typically, the residence time in each drum was just over 3 minutes, with a total treatment time after passing through three drum modules being around 10 minutes. If at this stage the sample was still contaminated, it was then recirculated. The novel reactor configuration exhibits certain features, which are important for the viability of the process in a large-scale application, these include the ability to operate the reactor in both batch and continuous mode. The use of Hombikat pellet catalyst removes the filtration problems associated with batch reactors; the reactor is constructed from Perspex, which has a high light penetration which leads to improved energy utilization. The reactor configuration also eliminates the need for oxygenation other than oxygen uptake from air through openings on both sides of the reaction vessels. The degradation of $\mathrm{MB}$ is reported under three sets of conditions (i) UV illumination alone, (ii) catalyst + UV illumination and (iii) catalyst without UV illumination. The wastewater sample was taken from an interceptor wastewater collecting effluent from a Garage Forecourt. This sample contained a mixture of hydrocarbons at a total COD level of between 3,500 and 4,000 $\mathrm{ppm}$. The destruction of the hydrocarbons was monitored by both measuring the chemical oxygen demand of the sample and also by gas chromatography/mass spectrometry (GCMS) using a Hewlett Packard model 5890 series IIGC connected to a Hewlett Packard model 5971A mass selective detector (Figure 13).

The COD of the water samples was also measured as an indicator of the total hydrocarbon level in the untreated and treated samples. Table 2 shows the mean COD values obtained from five experimental runs with each point being an average of three samples. It can be seen that the COD value decreases very quickly during the time it takes to process through the three consecutive reaction drums (10-minutes reaction time). As these experiments were performed on different days and the

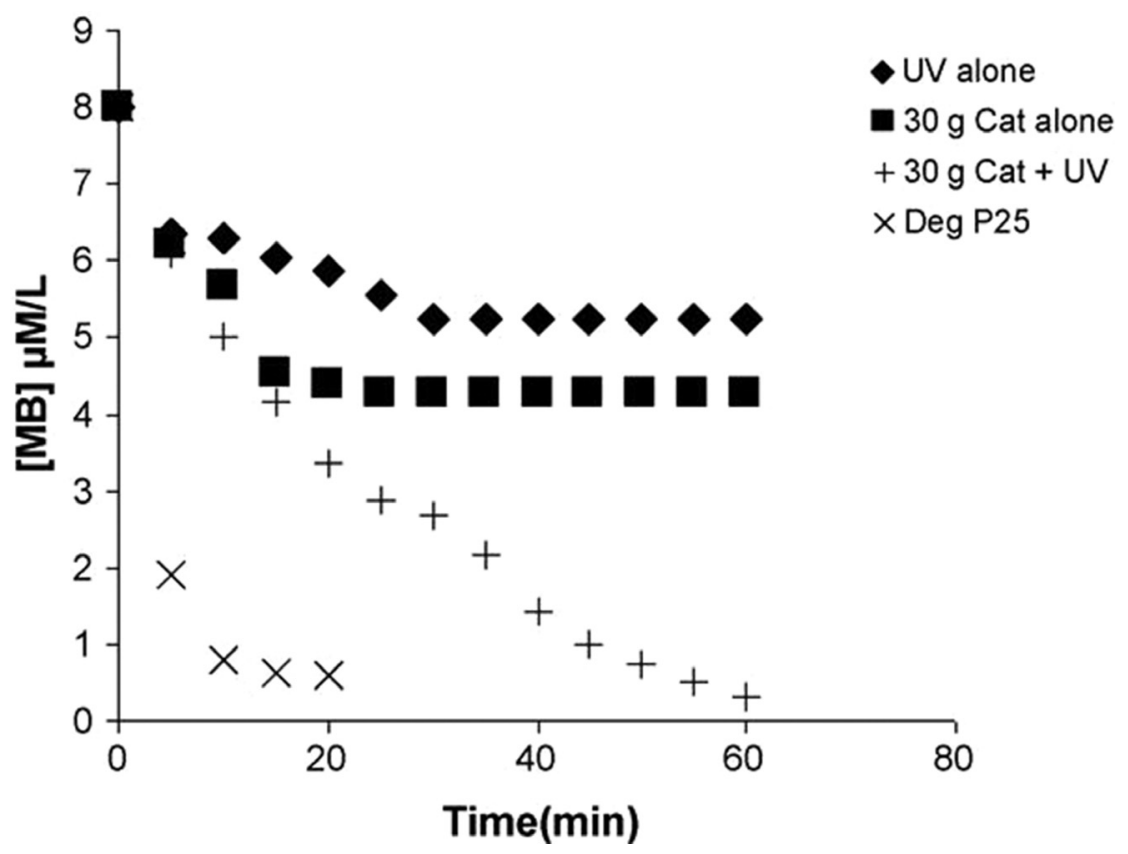

Figure 13 Methylene blue degradation by pelletised $\mathrm{TiO}_{2}$ (30 g Cat alone and $30 \mathrm{~g}$ Cat + UV) and Degussa P25 (1 g/L Deg P25) reproduced from [26] with permission from Elsevier. 
Table 2 Chemical oxygen demand values after continuous flow reaction through three reactor drums.

Sample pass

Mean COD mg/L

0 pass treatment

3,618

1st pass through drum reactor

2nd pass through drum reactor

2,166

868

Source: Reproduced from [9] with permission from Hindawi

wastewater effluent was decanted from a large storage drum, it is possible that the effluent content was not $100 \%$ consistent.

\section{Conclusion}

The results of this study have shown the development of a drum reactor through evolutionary designs, which proved effective for the removal of both dye and hydrocarbon contamination from wastewater effluents. With the development of the pelletised $\mathrm{TiO}_{2}$, it has been possible to develop an alternative reactor configuration with a smaller foot print. Conventional powder catalyst systems have traditionally posed removal problems downstream, requiring filtration and settling to remove powder from the effluent. This limits the type of reactor design to batch, as it is impractical to provide online filtration for a continuous flow reactor system.

The three photocatalytic reactor versions reported in this study were configured for batch and eventually continuous flow. From the initial single drum batch prototype through the three drum continuous flow unit, there has been a progressive increase in treatment capacity. From reactor version 1, it was shown that increased drum rotation speeds greatly increased the degradation rate of target compounds. This indicated the important relationship between catalyst agitation, catalyst-target interaction and photons reaching the catalyst surface.
Similarly with the illumination source position, the closer the light source was placed, the higher the degradation rate. This showed the correlation between photons reaching the catalyst and target degradation. Version 1 also highlighted a limitation of the baffle design as it caused the catalyst to gather towards one end of the drum. The longitudinal baffle, although excellent for mixing, did not counteract the mechanical corkscrew effect of rotation. To alleviate this effect, several different baffle arrangements were experimented with. Moving away from solid baffles and utilising individual baffles within the reactor initially showed promise, but the longitudinal arrangement still caused a corkscrew of the catalyst. A secondary baffle arrangement was examined that utilised a corkscrew pattern in an attempt to counteract the gather of catalyst. This proved unsuccessful. The desired stable mixing without a gathering of catalyst was found from a V pattern arrangement of smaller baffles. This was the design carried forward and reported in versions 2 and 3 and was also patented.

Investigations utilising version 3 , the drum continuous flow reactor, each drum contained the same quantity of catalyst, GC/MS results showed the effective 90\% removal of VOCs over 5 minutes. The mechanical mixing action of the paddle array within the reactor tubes greatly increases the pollutant-catalyst interface, enhances mass transport and also removes the need for additional air to be added to the system. Using a standard indicator for organic compounds in water, chemical oxygen demand, the drum reactor showed an $85 \%$ reduction of organic content.

It should be noted, as previously reported, that for the design of reactors reported here have been developed as a "Polishing" step and complementary technology to existing techniques. This technique of wastewater treatment limits the degree of contamination which can be processed, as the kinetics of the process would require very significant reaction times and the photocatalytic process cannot compete with existing technologies.

\section{References}

1. Fox MA, Dulay MT. Heterogeneous photocatalysis. Chem Rev 1993;93:341-57.

2. McCullagh C, Skillen N, Adams M, Robertson PK. Photocatalytic reactors for environmental remediation: a review. J Chem Tech Biotechnol 2011;86:1002-17.

3. Augugliaro V, Loddo V, Palmisano L, Schiavello M. Performance of heterogeneous photocatalytic systems: influence of operational variables on photoactivity of aqueous suspension of TiO2. J Catalysis 1995;153:32-40.

4. Mills A, Le Hunte S. An overview of semiconductor photocatalysis. J Photochem Photobiology A Chem 1997;108:1-35.

5. Ollis DF, Pelizzetti E, Serpone N. Photocatalyzed destruction of water contaminants. Environ Sci Technol 1991;25:1522-9. 
6. Chen D, Li F, Ray AK. External and internal mass transfer effect on photocatalytic degradation. Catalysis Today 2001;66:475-85.

7. Minero C, Vione D. A quantitative evalution of the photocatalytic performance of $\mathrm{TiO}_{2}$ slurries. Appl Catalysis B Environ 2006;67:257-69.

8. Robertson P, Campbell I, Russell D. Apparatus and method for treating a fluid by means of a transparent container. World Patent (W02005033016), 2006.

9. Adams M, Campbell I, Robertson PK. Novel photocatalytic reactor development for removal of hydrocarbons from water. Int J Photoenergy 2008;7.

10. Hoffman AJ, Lee H, Mills $G$ and Hoffman MR. Photoinitiated polymerization of methyl methacrylate using Q-sized zinc oxide colloids. J. Phys. Chem., 1992;96:5540-5546.

11. Hoffmann MR, Martin ST, Choi W and Bahnemann DW. Environmental. Applications of Semiconductor Photocatalysis. Chem. Rev. 1995;95:69-96.

12. Mills A, Davies RH and Worsley D. Purification of Water by Semiconductor Photocatalysis. Chem. Soc. Rev., 1993; 93:417-425.

13. Egerton TA and King CJ. The influence of light intensity on photoactivity in titanium dioxide pigmented systems. J. Oil Chem. Assoc. 1979;62:386-391.

14. Okamoto K, Yamamoto Y, Tanaka H, Tanaka M and Itaya A. Heterogeneous photocatalytic decomposition of phenol over $\mathrm{TiO}_{2}$ powder. Bull. Chem. Soc. Jpn., 1985;58:2015-2022.

15. Martin ST, Herrmann H, Choi W and Hoffmann MR. Time-resolved microwave conductivity. $\mathrm{TiO}_{2}$ photoreactivity and size quantization., Trans. Faraday Soc. 1994;90:33153322.

16. Kormann C, Bahnemann DW and Hoffmann MR. Photolysis of Chloroform and Other Organic Molecules in Aqueous $\mathrm{TiO}_{2}$ Suspensions. Environ. Sci. Technol. 1991;25:494-500.

17. Jaeger $C D$ and Bard AJ. Spin trapping and electron spin resonance detection of radical intermediates in the photodecomposition of water at $\mathrm{TiO}_{2}$ particulate systems. J. Phys. Chem., 1979;83:3140-3152.

18. Wang $Y$ and Hong C-S. Effect of hydrogen peroxide, periodate and persulfate on photocatalysis of 2-chlorobiphenyl in aqueous $\mathrm{TiO}_{2}$ suspensions. Wat. Res., 1999;33:2031-2036.

19. Crittenden JC, Hu S, Hand DW and Green SA. A kinetic model for $\mathrm{H} 2 \mathrm{O} 2 / \mathrm{UV}$ process in a completely mixed batch reactor. Wat. Res., 1999;33:2315-2328.

20. Cornish BJ, Lawton LA, Robertson PK. Hydrogen peroxide enhanced photocatalytic oxidation of microcystin-LR using titanium dioxide. Appl Catalysis B Environ 2000;25:59-67.

21. Lakshmi S, Renganathan $\mathrm{R}$, Fujita S. Study on $\mathrm{TiO}_{2}$-mediated photocatalytic degradation of methylene blue. J Photochem Photobiology A Chem 1995;88:163-7.

22. Marletta MA. The merck index. An encyclopedia of chemicals, drugs, and biologicals. Tenth Edition. Martha Windholz, Rahway, New Jersey: Merck \& Co., Inc., 1983. Hepatology 1985;5.

23. Zhang T, Oyama T, Aoshima A, Hidaka H, Zhao J, Serpone N. Photooxidative $\mathrm{N}$-demethylation of methylene blue in aqueous $\mathrm{TiO}_{2}$ dispersions under UV irradiation. J Photochem Photobiology A Chem 2001;140:163-172.

24. Otsuka-Yao-Matsuo S, Ueda M. Visible light-induced photobleaching of methylene blue aqueous solution using $(\mathrm{Sr} 1-\mathrm{xLax}) \mathrm{TiO}_{3}+\delta-\mathrm{TiO}_{2}$ composite powder. J Photochem Photobiology A Chem 2004;168:1-6.

25. Zhang T, Oyama TK, Horikoshi S, Hidaka H, Zhao J, Serpone N. Photocatalyzed N-demethylation and degradation of methylene blue in titania dispersions exposed to concentrated sunlight. Solar Energy Mater Solar Cells 2002;73:287-303.

26. McCullagh C, Robertson PKJ, Adams M, Pollard PM, Mohammed A. Development of a slurry continuous flow reactor for photocatalytic treatment of waste water. J Photochem. Photobiology A: Chem 2010;211:42-46. 